\title{
Deporte y entrenamiento deportivo como alternativas frente a la "degeneración de la raza" durante la primera mitad del siglo XX en Colombia*
}

\author{
Jair Hernando Álvarez Torres * \\ José Tomás Cortés Díaz ${ }^{* *}$
}

Recibido: 25 de mayo del 2018 Enviado a pares evaluadores: 18 de junio del 2018 Aprobado por Comité editorial: 29 de junio del 2018 https://doi.org/10.22395/csye.v7n13a4

\section{RESUMEN}

El deporte y el entrenamiento deportivo han sido prácticas pensadas en diferentes coordenadas del planeta, sin embargo, sus modelos permanecieron anclados al contexto cultural europeo hasta tiempos recientes. Este artículo se concentra en la descripción analítica de algunos condicionantes de la época que promovieron la propagación del juego, el deporte y el entrenamiento deportivo como alternativas frente a la pregunta sobre la decadencia de la cultura colombiana y la degeneración de la raza, en particular a principios del siglo $\mathrm{XX}$ en Colombia, la cual postularon los intelectuales de la Generación del centenario, quienes procuraban un proyecto modernizador del país. El análisis documental realizado sugiere que este grupo de intelectuales elitistas tenía los pies en Colombia, pero su pensamiento estaba en Europa y Estados Unidos, y querían ver o convertir cultural y socialmente a Colombia en un país del viejo continente.

Palabras clave: biopolítica; deporte; entrenamiento deportivo; educación física.

Artículo de revisión y análisis, producto del ejercicio académico de los autores. A través de este texto se intenta articular el tema del entrenamiento deportivo y su papel en el proceso biopolítico en Colombia. Para ello, se alimenta de cada uno de los saberes de sus autores.

** Doctor en Historia de la Universidad Nacional de Colombia. Licenciado en Filosofía de la Universidad de Antioquia (Colombia). Con estudios Doctorales en Historia en la Universidad Torcuato Di Tella (Argentina). Profesor de la Facultad de Ciencias Sociales y Humanas de la Universidad de Medellín (Colombia). Correo electrónico: jhalvarez@udem.edu.co

*** Magíster en Educación de la Universidad de San Buenaventura, sede Medellín (Colombia). Especialista en Entrenamiento Deportivo de la Universidad de Antioquia (Colombia) y Licenciado en Educación Física de la misma universidad. Tecnólogo Deportivo del Politécnico Colombiano Jaime Isaza Cadavid (Colombia). Director del programa de Actividad Física y Deporte de la Universidad Católica Luis Amigó. Correo electrónico: jotomas2001@yahoo.com.ar 


\section{Sports and Training seen as Alternatives to "Race Degeneration" during the first half of the $\mathbf{2 0}^{\text {th }}$ Century in Colombia}

\section{ABSTRACT}

Sport and training are practices that have been thought and elaborated in different coordinates of the planet, however, their models remained anchored to the European cultural context until recent times. This article focuses on the analytical description of some conditioning factors of such times that promoted games, sports and training as possible solutions for the so-called decline of Colombian culture and race degeneration, particularly in the early 20th century. Intellectuals belonging to the
Centennial Generation who sought a modernizing project for the country declared the race degeneration and started such debate. Literature review suggests that this group of elitist intellectuals had their feet in Colombia, but their perspectives and understandings focused in Europe and the United States; they sought to transform Colombia -culturally and socially-into a country of the old continent.

Keywords: biopolitics; sports; training; physical education.

\section{Esporte e treinamento esportivo como alternativas à "degenepação da raça" durante a primeira metade do século XX na Colômbia}

\section{RESUMO}

O esporte e o treinamento esportivo foram práticas pensadas em diferentes coordenadas do planeta, no entanto, seus modelos permaneceram ancorados no contexto cultural europeu até tempos recentes. Este artigo se concentra na descrição analítica de alguns condicionantes da época que promoveram a propagação do jogo, do esporte e do treinamento esportivo como alternativas diante da pergunta sobre como a decadência da cultura colombiana e da degeneração da raça, em particular no início do século XX na Colômbia, que foi postulada por intelectuais da Geração do centenário que buscavam um projeto modernizado do país. A análise documental realizada sugere que esse grupo de intelectuais elitistas estavam com os pés na Colômbia, mas seu pensamento estava na Europa e nos Estados Unidos e queriam ver a Colômbia convertida, cultural e socialmente, em um país do velho continente.

Palavras-chave: biopolítica; esporte; treinamento esportivo; educação física. 


\section{Introducción}

El entrenamiento deportivo, si bien lo practica un individuo en concreto o grupos de individuos diferenciados, lleva intrínseco un modo de racionalidad que se le pretende imponer a la población, tal como sucedió en la primera mitad del siglo XX, a propósito de los debates sobre el progreso y la raza en Colombia. No obstante, este fenómeno no comenzó de manera exclusiva en dicho contexto, al contrario, el deporte y el entrenamiento deportivo son prácticas pensadas en diferentes coordenadas del planeta, pero sus grandes modelos han estado anclados al contexto cultural europeo, tal como se expone en la primera parte de este texto.

Este artículo, se concentra en la descripción de algunas condiciones de la época que permitieron la propagación del juego, el deporte y el entrenamiento deportivo como alternativas frente a la preocupación por la decadencia de la cultura colombiana y la degeneración de la raza en particular. Los intelectuales de principios del siglo XX, especialmente aquellos a los que se denomina Generación del Centenario ${ }^{1}$, pretendieron determinar la causa de dicha degeneración y las formas de profilaxis adecuadas para su atenuación y/o resolución, con explicaciones de tipo biológico-ambientales, religiosas y morales. Estos personajes tenían en común que:

[...] habían sido formados o tenían contacto permanente tanto con el norte del continente americano como con el viejo continente, hasta que estalla la Primera Guerra Mundial y aquellos no pueden continuar sus viajes al extranjero, y al tener que quedarse en Colombia, empiezan a sentir cierto desconsuelo ante la situación de atraso que vivía el país. De esta manera, nos atrevemos a creer que este grupo de intelectuales elitistas tenía los pies en Colombia pero su pensamiento estaba en Europa y Estados Unidos, o mejor dicho, querían ver o convertir cultural y socialmente a Colombia en un país más del viejo continente. (Álvarez, 2012, p. 53)

Resulta de gran relevancia para este trabajo la idea de estos intelectuales de que dicha degeneración se originaba ante la ausencia de una educación o cultura física en los colombianos de la época, tal como lo expuso el médico psiquiatra Miguel Jiménez López. Este autor, por ejemplo, consideraba que la enseñanza secundaria era incompleta, ya que se dedicaba la mayoría del tiempo al intelecto y la memoria, a expensas de la formación del carácter y del cuerpo. Su propuesta apuntaba a una reforma educativa que se adaptara a las realidades del país y de la vida, en concordancia con los preceptos de la "escuela activa" (Jiménez

1 Entre otros, destacan los siguientes: Lucas Caballero, Jorge Bejarano, Emilio Robledo, Alfonso Castro, Simón Araujo, Calixto Torres Umaña, Miguel Jiménez López, Luis López de Mesa y Agustín Nieto Caballero. Este apodo se les otorgó gracias a la coincidencia entre la celebración del Primer Centenario de la Independencia de Colombia y los destacados aportes intelectuales que realizaon desde los cargos públicos y el ejercicio de sus profesiones a nivel social. 
López 1928). La degeneración de la raza tendría de esta forma una posible causa y etiología en el abandono de la educación y la cultura física, puesto que,

[...] no hay exageración al establecer este lamentable balance de la vida escolar. Si muchas veces se cree hallar causas distintas de ésta [sic] a la debilidad creciente de las generaciones nuevas, es porque se quiere perder de vista el más poderoso factor de degeneración: el abandono de la educación física. (Jiménez López, 1928, p. 228)

En el caso de López de Mesa, Aline Helg dice lo siguiente:

López de Mesa tenía una visión determinista del pueblo colombiano. En su Introducción a la historia de la cultura en Colombia (1930), comparaba esquemáticamente la psicología del negro con la de un niño despreocupado y la del indio con la de un anciano malicioso. La superioridad de la raza española, y en particular la de los nórdicos, le parecía evidente. Sin embargo, según él, todos tenían las capacidades intelectuales y físicas que una educación apropiada y un programa de higiene nacional permitirían desarrollar. Uno de los problemas cruciales de Colombia era la fosa que separaba el campo de la ciudad. (Helg, 2001, p. 152)

De todas maneras, dicha afirmación dejó en claro que para la época, había ciertas falencias en la instrucción pública colombiana, y que de una u otra forma, todos los intelectuales estaban preocupados por darle la mejor solución posible a este asunto a favor del progreso y la civilización del pueblo colombiano, y algunos se atrevieron a plantear que el problema de la raza era un problema de la formación y no un asunto filo u ontogenético, es decir, que más bien era un problema de tipo cultural y no versaba sobre un perfil biológico. En todo caso, se plantearon propuestas que permitieran la conducción de los comportamientos de los cuerpos desde su vida cotidiana, es decir, una vivencia de la biopolítica bajo otras condiciones, o en palabras de Giddens y Fehér,

[...] el punto de la biopolítica es la vida cotidiana, y sus olas reverberan desde allí en círculos constantemente crecientes hacia el centro del dominio público. Esto es evidente por sí mismo puesto que los problemas de "el cuerpo" se articulan "naturalmente" en la vida diaria. (1995, p. 50)

De este modo, una nueva estrategia biopolítica ${ }^{2}$ comienza a ubicarse en las aulas de clase para impactar la vida social y cultural de los sujetos. La educación física, y su relación con el cuerpo, el movimiento, el juego y el ocio, tendrán inje-

2 Es ineludible mencionar el compromiso inicial de este artículo con la toma de posición frente a la forma como ha de entenderse el concepto de biopolítica. Por eso, se asume este concepto a partir de la siguiente definición: "Hay que entender por biopolítica la manera en que, a partir del siglo XVIII, se buscó racionalizar los problemas planteados a la práctica gubernamental por los fenómenos propios de un conjunto de vivientes en cuanto población: salud, higiene, natalidad, longevidad y raza" (Castro, 2004, p. 45). Se reconoce además, que a partir del ejercicio de poder, la biopolítica se dedica al cuerpo múltiple o colectivo, que se diferencia en cierta medida de los procesos de disciplinamiento logrados a partir de un ejercicio de poder sobre el cuerpo individual, que si bien es ejercido sobre un sujeto en particular, hará parte de ese cuerpo múltiple que también será gobernado. 
rencia directa con los procesos de modernización a través del control del tiempo, la dinamización de la escuela activa, los juegos recreacionales, el fortalecimiento de la raza y la organización del deporte, siendo este último el lugar dentro del cual se ubica el entrenamiento deportivo como centro de interés de este trabajo.

\section{El entrenamiento deportivo}

El entrenamiento deportivo como área específica de la educación física, comparte los mismos principios formativos y antropológicos de la educación. No obstante, en su proceso para constituirse como un saber especifico, transitó por varias etapas, producto de las transformaciones que se daban al interior de la educación física. Al revisar la historia, se podría preguntar ¿Qué estrategias o técnicas aplicaron los pedagogos para incrementar el rendimiento humano, y esto cómo los llevo a transformar los cuerpos? Para ayudar en esta tarea, las diversas escuelas de gimnasia presentan poderosos sistemas de ejercicios, los cuales ordenan de forma progresiva y seriada que, además, permitieron pasar de una educación física basada en procedimientos heterogéneos a un sistema progresivo en el cual las tareas y actividades se analizaban cuidadosamente. En este momento, el término "entrenamiento" ya tenía una aplicación destinada a cultivar y promover las acciones técnicas específicas de la educación física (Rauch, 1985).

A lo largo del tiempo, los conceptos teóricos del entrenamiento deportivo han sobrellevado diversas transformaciones en tanto que saberes específicos, con relación al momento histórico, ubicación geográfica y pensamiento de quien lo aplica. Ello permite recoger planteamientos y catalogarlos en dos corrientes a propósito de su relación con el concepto de educación: una que lo ubica como una cuestión de desarrollo biológico-pedagógico y otra como solo biológico. Para los teóricos del entrenamiento deportivo que reducen el entrenamiento deportivo a una situación de adaptación biológica, tales como González Badillo (2002), Ozolin, Grosser, Starischka y Zimmermann (1983), Platonov (1988), Zimkin y Manno (1991), todos ellos citados por Pérez, J. y Pérez, D. (2009), el entrenamiento deportivo es una cuestión técnica llevada a cabo con procedimientos instrumentales e internalistas en los cuales solo es importante dosificar esfuerzos de forma creciente y continúa para generar economía funcional.

La otra corriente de pensamiento, para la cual el entrenamiento deportivo supone una relación entre lo biológico y lo pedagógico, está representada por autores como Martin (1977), Matveiev, Bompa y Vittori (1983), Verkhoschansky (1985), Zintl (1991), Quezada (1997), Pérez y el colectivo de profesores del departamento de Educación Física de la Universidad Pedagógica Nacional de Colombia (2008), también citados por Pérez, J. y Pérez, D. (2009). Estos coinciden 
al afirmar que el entrenamiento deportivo es un proceso complejo que aumenta las condiciones funcionales, personales y sociales de los individuos. De hecho, se propone el entrenamiento deportivo como un proceso encaminado a la formación multilateral del individuo, desde el punto de vista físico-funcional, motriz, psicológico, cognitivo, social y moral; estas relaciones se encuentran reguladas por las mediaciones que se establecen entre el entrenador-pedagogo y el alumno-atleta. Por esto, la necesidad de un tratamiento particular en relación a las características formativas que se dan a través del acto educativo que allí se configura.

Para entrar en el tema del entrenamiento deportivo en Colombia y su papel como estrategia biopolítica, se hace necesario hacer un recorrido por su proceso histórico. Es posibles estudiar la evolución del rendimiento deportivo con cierta seguridad y fidelidad desde aproximadamente finales del siglo XIX. Previo a ese momento, los deportistas entrenaban basados en carreras de larga duración, de forma continua y uniforme, sin una planificación sistemática de las tareas a desarrollar. Sin embargo, los antecedentes dan cuenta que el proceso partió de los métodos de entrenamiento. De hecho, los primeros procedimientos tenían una fundamentación empírica, pero fueron evolucionando hasta convertirse en métodos caracterizados por la implementación de principios didácticos; se empezó a dotar de sentido la ejecución de ejercicios y los procedimientos para cada ejecución, aparecieron las escalas de valor para las actividades desarrolladas, como ejercicios simples, complejos, de preparación, competitivos, aplicados, entre otros. También, desde la aplicación del método se reconocieron y nombraron los efectos del entrenamiento sobre el organismo (Rauch, 1985).

Para inicios del siglo XX el método de entrenamiento consistía en correr distancias iguales o muy cercanas a la competencia, así como al ritmo que se pretendía mantener en ella; se empezó a sustituir el método de entrenamiento continuo por un tipo de entrenamiento alterno, en el cual los tramos y las repeticiones de ejercicios ya no se hacían a una velocidad uniforme e ininterrumpida (Forteza de la Rosa, 1999)), sino que eran intercalados con cierto número de aceleraciones. Sin embargo, el entrenamiento carecía de fundamentación científica, por lo tanto no era planeado de manera sistemática; aunque se le prestaba especial importancia al cuidado personal y la dieta, la base del entrenamiento eran las carreras y los ejercicios gimnásticos generales. Ahora bien, el principio básico era entrenar con más duración que la especialidad competitiva. Durante este periodo, se dio una marcada influencia de las diferentes escuelas aplicadas en la educación física y producto de ello se empezó a generar una preocupación por la salud de los individuos que entrenan. De dicha preocupación, se dedujo que el deporte, a pesar de tener un fundamento competitivo para su entrenamiento, también tenía un principio educativo y esto quedaba manifiesto en la 
preocupación por el cuidado del cuerpo como principio fundamental para la práctica deportiva.

Después de las Olimpiadas de Estocolmo (1912), los entrenamientos fueron más ordenados, y a partir de allí se empezaron a sistematizar los datos prácticos y dispersos sobre el tema, dándole al trabajo empírico un giro y encaminándolo a un trabajo más planificado. El resultado fue un mayor volumen para la preparación, de tal forma que en la temporada competitiva el rendimiento fuese estable, esto llevó a los atletas destacados a dividir el entrenamiento en dos sesiones diarias. Se hacía mucho énfasis en la técnica de ejecución, por lo cual se incorporaban activamente múltiples ejercicios preparatorios en el entrenamiento. Es entonces, el periodo comprendido entre 1912 y la mitad del siglo XX en que inicia la construcción del campo epistemológico del entrenamiento deportivo.

El finlandés Laury Pinkala destaca como pionero de la sistematización, pues introduce novedades como trotes lentos hasta sprint y pausas parciales no totales (de 10-15 s). Al igual que Matsusawa en Japón, entrenador de natación, por sus trabajos de flexibilidad y la técnica de recuperación. En Suecia en 1930, Gosse Holmer creó el sistema Fartlek (fart: velocidad, y lek: juego) y sustentó su teoría en la importancia que se le debe dar, tanto al trabajo como al descanso. Sin embargo, el promotor de este método fue Gosta Olander, partidario del trabajo en contacto con la naturaleza. Su método es duro y exigente, seguido por tareas suaves y respiratorias. Para Olander, el entrenamiento no supone solamente un esfuerzo corporal, sino también una preparación mental, su idea fundamental era que las verdaderas fuentes del esfuerzo físico no están en los músculos sino en el cerebro. En Gosse Holmer y Olander, hay una clara contraposición a la postulación de Pinkala (trabajo corto pero intenso), pues privilegian un esfuerzo mediano y extenso, es decir que se inclinan por el factor cuantitativo.

En el caso Húngaro, Mihaly Igloi adaptó los métodos de entrenamiento de los finlandeses y los hizo conocer, pero con ciertas "adaptaciones": una gran dosis de trabajo diario $(20-40 \mathrm{Km})$, el respeto por la individualidad, el trabajo centrado en los "puntos fuertes" de cada atleta, gran variedad del entrenamiento fraccionado y la no repetición de dos sesiones iguales para un mismo atleta. En 1940, el Alemán Tony Nett generó una sistematización del entrenamiento deportivo mediante el ordenamiento de los métodos y sistemas existentes. Nett los clasificó de acuerdo con los objetivos, organizó la temporada anual, escribió sobre el entrenamiento fraccionado y otros temas.

Se deben destacar los importantes aportes de Holmer y Olander, a través de sus métodos de trabajo, situaron la capacidad del sujeto por encima de cualquier modelo matemático de carga, es decir, los sujetos entrenaban siempre y cuando su capacidad de trabajo individual lo permitiera, sin llegar a límites de 
sobrecarga exagerada. Lo anterior supone una preocupación por el bienestar del individuo, además, se empiezan a manejar los conceptos de preparación mental e individualidad, lo que lleva a pensar el deporte de competencia desde otra lógica en su preparación y competencia.

Si bien se intenta hacer una descripción de la forma en la que se adquiría el rendimiento para inicios del siglo XX, esto no constituye en sí una teoría del entrenamiento, eran métodos particulares que daban resultados en diferentes partes de mundo. Para este momento, el término "record" no era sustancial, porque no se llevaban registros de tiempos y marcas anteriores, solo importaba ganar Forteza de la Rosa (1999).

Todo esto era completamente desconocido en el contexto colombiano, ni siquiera se había planteado un modelo de rendimiento al interior del país. Pero a partir de inicios del siglo XX y bajo la presión ejercida por la pobreza que deja la Guerra de los Mil Días, empieza en Colombia todo un movimiento para el disciplinamiento y la formación corporal, que al final terminaría fundando las bases de los procesos de rendimiento en el país. La Guerra de los Mil Días (que en realidad fueron 1.100 días) se dio por el conflicto entre conservadores y liberales. El país estaba gobernado por los conservadores, pero los liberales ejercían una fuerte presión al poder, lo que derivó en la declaración de guerra civil por parte de los liberales en 1899; fue una época intensa que dejo gran cantidad de muertos, cerca de cien mil vidas se perdieron en todo el territorio nacional.

Como era de esperarse, el resultado de la guerra fue un país devastado, con altos niveles de pobreza, sin vías de comunicación, con carreteras destruidas y una deuda externa e interna considerable. Pero, lo más representativo que dejó la guerra fueron las preocupantes condiciones de enfermedad de la población. Para los intelectuales de la época, esta era una situación que tenía que corregirse de forma inmediata para poder pensar en la reconstrucción del país ${ }^{3}$. Una reflexión seria en torno a lo que se debería hacer para regenerar la raza en Colombia resultaba inaplazable.

3 El diagnóstico de los intelectuales era que se tenía una población desordenada, sin control social y enferma. Concluyeron entonces que con esta población no era posible llevar a cabo el proyecto modernizador en Colombia. Así, el tema de la raza que imperó en el país durante las dos primeras décadas del siglo XX clasificó a la sociedad en aptos y no aptos, de tal forma que, desde la educación corporal (en este caso la educación física), se implementó un discurso para fortalecer el modelo económico, social y político de la época. Algunos intelectuales como Miguel Jiménez López, Luis López de Mesa, Jorge Bejarano y Lucas Caballero, llegaron al consenso de que la población colombiana era incapaz de desarrollar el proyecto modernizador (Ruiz Patiño, 2009). Para esta afirmación se dieron a la tarea de hacer una clasificación de las características de la población, definiendo los rasgos corporales, fisiológicos y actitudinales que deberían tener aquellos individuos que podrían brindarle al país su fuerza de trabajo. Para el pensamiento de la época, se requería la formación de un hombre fuerte, vigoroso y sano para que sirviera a la economía y la protección del Estado (Cortes Díaz, 2015, p. 74). 


\section{El deporte y el entrenamiento deportivo en Colombia durante la primera mitad del siglo XX}

Como se mencionó anteriormente, para algunos intelectuales colombianos de la época, también conocidos como la Generación del centenario, el debate sobre la raza tuvo fuertes repercusiones en la concepción educativa y formativa del pueblo colombiano en lo concerniente al ideal de hombre a formarse en las escuelas. A continuación se hace mención de algunos de ellos con sus planteamientos respectivos.

Lucas Caballero: negó la gravedad de la miseria del pueblo colombiano con el pretexto de que el pobre no podía desear lo que no conocía, y preconizó como solución al subdesarrollo la generalización de la enseñanza elemental. Jorge Bejarano: higienista de profesión, vio en la raza negra el principal factor de regeneración de los pueblos mestizos e indígenas montañeses. Emilio Robledo y Alfonso Castro: estos médicos antioqueños recusaron las acusaciones de degeneración colectiva afirmando que los locos no eran tan numerosos en Colombia como en los países europeos y que las causas de criminalidad y de locura se encontraban en las enfermedades, el alcohol y la sífilis, mas no en las razas. Simón Araujo: su tesis se centraba en la educación, sostuvo que la juventud colombiana tenía dificultades intelectuales iguales a las de otras naciones, pero que había perdido su entusiasmo por los estudios a causa de la errada política escolar del Ministerio de Instrucción Pública; estimaba igualmente que uno de los mayores problemas de Colombia era su pobreza, la cual le impedía desarrollarse.

Calixto Torres Umaña: se inclinó por definir la debilidad de la raza colombiana a partir de elementos biológicos. Miguel Jiménez López: fue quien comenzó el debate en torno a la degeneración de la raza con una ponencia en 1918 presentada en el Tercer Congreso Nacional de Médicos. La ponencia se denominaba "Nuestras razas decaen. El deber actual de la ciencia". Su tesis se centraba en afirmar que una degeneración colectiva afectaba al pueblo colombiano, tanto en lo físico como en lo psíquico. La inferioridad fisiológica del pueblo mal alimentado se notaba en la pequeña estatura, su cantidad de glóbulos rojos y su temperatura corporal, inferiores a la normal. En cuanto a lo psíquico, consideraba que la raza débil colombiana se debía a la mezcla entre los colonizadores españoles, los cuales eran aventureros inmorales, y los indígenas, y que esto se evidenciaba en el aumento de la locura y la criminalidad, la frecuencia de guerras civiles, el recurso al suicidio, el alcoholismo y la sífilis. Luis López de Mesa: estaba de acuerdo con la perspectiva pesimista de Jiménez López acerca de la herencia racial negativa del pueblo colombiano (Helg, 2001, pp. 111-115).

En este artículo, nos ocuparemos de la forma en la que el deporte comienza a tener un rol protagónico en el escenario social y su relevancia para el proyecto 
modernizador del país. Para muchos de los intelectuales de principio del siglo $\mathrm{XX}$ en Colombia, el cuerpo surge como instrumento de posibilidades para la salvación y el futuro del país, como cuerpo sano, vigoroso, disciplinado, saludable, controlado, educado, estético, distinto, bien puesto, ejercitado, rendidor, resistente, etc. (Runge Peña y Muñoz Gaviria, 2012, p. 83). Asimismo, la falta de control social e higiene personal eran consideradas foco de muchas enfermedades, así como la causa del atraso social y económico del país. Para resolver esto, fue la educación, y específicamente la educación física, la encargada de dar soporte a la educación del cuerpo y mejorar la higiene de la población.

Pero más allá de los esfuerzos académicos e intelectuales, se requería de unas condiciones que optimizaran esos esfuerzos, lo cual se comienza a materializar a partir de tres sucesos importantes para la primera mitad del siglo $\mathrm{XX}$, a propósito del deporte como estrategia para combatir la degeneración de la raza: 1) La sanción de la Ley 39 de 1903, Ley orgánica de la educación y su Decreto reglamentario 491 de 1904; 2) La sanción de la Ley 80 de 1925, que reglamenta la educación física y los deportes; y 3) La creación en 1936 del Comité Olímpico Colombiano, cuya misión era apoyar los procesos olímpicos y brindarle al deporte y, en especial al alto rendimiento, una nueva visión y estructura para su desarrollo. El interés de este trabajo se centra en estos tres aspectos, los cuales, poco a poco, fueron abriendo camino para la consolidación del campo deportivo en Colombia.

De acuerdo con los trabajos realizados por algunos investigadores en Colombia, la primera mitad del siglo $\mathrm{XX}^{4}$ se caracterizó por una serie de cambios en los epicentros educativos de Colombia. Prueba de lo anterior fue, por ejemplo, la fundación del Gimnasio Moderno, un colegio en el que se experimentó con la pedagogía reformista o activa ${ }^{5}$ como manera de formar moral y físicamente a sus

4 Nos referimos precisamente a la producción historiográfica que se ha hecho de la historia de la educación en Colombia, tal como sucede con los trabajos de autores como: Le Bot (1979) que se concentra en la relación entre Estado, educación y clases sociales; Bohórquez Casallas (1956) el cual ha hecho un aporte significativo sobre la división en cuatro grandes periodos de la educación en el territorio colombiano a partir de la escuela dominante: prehistóricas y primitiva (escuela espontánea o cósmica); hispano-granadina, de 1500 a 1820 (se combina la Escuela oral y verbalista con la Escuela memorista); democrático-moderna, 1820-1886 (Escuela objetiva); y democrática-contemporánea, 1886 a hoy (Escuela activa). El trabajo de Cacua Prada (1997) que impacta precisamente por su desconocimiento frente a la producción investigativa en historia de la educación y la pedagogía en Colombia durante las últimas dos décadas del siglo XX; Sáenz Obregón, Saldarriaga y Ospina (1997); y finalmente, el impresionante trabajo realizado por Helg (2001), texto de ineludible referencia cuando se trata de historiar la primera mitad del siglo XX en Colombia, pues no se queda en el mero análisis de las políticas educativas de la época, sino que recoge elementos periféricos y los vuelve importantes, tal como acontece con las discusiones de los intelectuales de la época con respecto al tema de la degeneración de la raza en Colombia, entre otros.

5 El movimiento de pedagogía de la reforma o activa representó uno de los momentos más ricos y productivos de la pedagogía en la historia y, particularmente, en Colombia. En el seno de este movimiento se desarrollaron numerosas ideas y planteamientos pedagógicos novedosos y abundaron las propuestas y proyectos teórico-prácticos. La importancia de este movimiento de comienzos del siglo XX radica en haber roto con la 
estudiantes. En concordancia con los planteamientos e ideas orientadoras del movimiento reformista o de pedagogía activa de comienzos de siglo, la pedagogía en Colombia cambió su mirada frente al sujeto en formación y reivindicó, como premisa fundamental, la actividad del estudiante ${ }^{6}$.

En lo que concierne a la educación física, desde la segunda década del siglo XX en el país se insistió en su intensificación y modernización, constituyéndose en uno de los pocos elementos de la nueva pedagogía que en Colombia logró un desarrollo sostenido e ininterrumpido (Sáenz, Saldarriaga, Ospina, 1997). La Ley 39 de 1903 y su Decreto Reglamentario 491 de 1904 oficializan la importancia de la educación física, la recreación y los deportes para la población colombiana, aunque al comienzo de la implementación de este marco jurídico en las escuelas, el ejercicio físico estuvo fuertemente focalizado en los varones, dado que los primeros instructores de educación física en las escuelas eran exmilitares del ejército, que tenían programado todo un régimen disciplinario para los cuerpos masculinos.

Fueron entonces estos instructores quienes comenzaron a implementar dicho marco jurídico, organizando juegos gimnásticos y paseo higiénico y recreativo durante una media jornada cada dos semanas, tal como reza en el artículo 63 del Decreto 491 de 1904: "Cada dos semanas se destinará medio día a paseo higiénico y recreativo. El institutor organizará juegos gimnásticos entre los alumnos". Esta idea fue fuertemente difundida y reconfigurada a través de las escuelas, con cada vez mayor énfasis e importancia, dado que los discursos médicos aplaudían este tipo de actividades por el bien de la población colombiana, y en especial, de las futuras generaciones. Es así que en la década de 1920 se da una proliferación de propuestas sobre la formación del cuerpo y su importancia social.

Por su parte, la Ley 80 de 1925 creó, entre otras cosas, la Comisión Nacional de Educación Física para combatir las causas de deterioro físico de la infancia,

concepción tradicionalista, pasiva y jerárquico-autoritaria de la educación, haber asumido una posición crítica frente a la "vieja escuela" (escuela tradicional) y haber propuesto una nueva manera de mirar la infancia y su educación, basada ahora en el respeto por el niño y en el fomento de su formación mediante la actividad. Dentro de los pedagogos que encarnaron esta nueva visión de la educación se encuentran, entre otros, Ovide Decroly (1871-1932), John Dewey (1859-1952), William James (1842-1910), Peter Petersen (1884-1952), Rudolf Steiner (1861-1925), Ellen Key (1849-1926), María Montessori (1870-1952), Helen Parkhurst (1887-1973), Célestin Freinet (1896-1966). No debemos olvidar que en las bases -antropológicas, políticas, pedagógicas- de este movimiento se encuentran implícita o explícitamente las ideas del precursor de esta nueva visión del niño y de su educación, a saber: Jean-Jacques Rousseau.

6 Por eso, a pesar del pluralismo teórico y experiencial, el movimiento de pedagogía de la reforma o activa pudo consolidarse gracias a una serie de ideas orientadoras en las que el niño entró a cumplir un papel determinante. De allí precisamente el término "paidocentrismo" (escuela, educación y contenidos ajustados y centrados en el niño, en sus intereses y necesidades) para caracterizar de un modo general a este movimiento, con sus diferentes orientaciones, variantes, tendencias, enfoques y respectivos modelos y aplicaciones. 
diseñando concursos y organizando un plan nacional de educación física obligatoria en las escuelas de educación primaria, secundaria y en las universidades. Durante la primera mitad del siglo XX, se realizaron juegos nacionales, torneos, e incluso, se reorganizó el Ministerio de Educación al crear la sección de Educación Física.

De igual modo, por medio del Decreto 1528 de 1936, se creó el Instituto Nacional de Educación Física como dependencia directa del Ministerio de Educación Nacional y posteriormente, de la Universidad Pedagógica Nacional, para la formación de profesores y de maestros de escuela primaria en ejercicio, por medio de cursos especiales. Los planes de estudio de dichos cursos se centraban en las prácticas y los saberes de la pedagogía experimental y el examen escolar: psicología, anatomía, fisiología, bioquímica, biología, nutrición y dietética, primeros auxilios y puericultura para las mujeres. Posteriormente, en 1937, se hizo énfasis en la dimensión psicológica del examen escolar al introducir las prácticas de la antropometría, los test de inteligencia, el diagnóstico y medición de las aptitudes y la orientación profesional.

La sanción de la Ley 80 de 1925, le dio el espaldarazo oficial a las intenciones científicas de los médicos, psiquiatras, sociólogos y pedagogos al propiciar una pedagogía activa en la escuela a través de la cual se instauró la reglamentación de la educación física y los deportes. Tal como lo plantean Runge, Muñoz y Álvarez (2005, p.124):

[...] la propuesta sería la defensa de la educación física, tematizada y perfeccionada por los sabios y las corporaciones científicas, como estrategia pedagógica para formar la voluntad y encaminar las energías hacia la realización de los ideales de hombre propios de la época, y donde se establece una relación directa entre cuerpo y moral para luchar contra la degeneración de la raza.

Por otro lado, dicha Ley creó la Comisión Nacional de Educación Física, compuesta por tres miembros nombrados por el poder ejecutivo, del Director General de Higiene y del Ministro de Instrucción Pública.

\footnotetext{
Algunas de sus funciones principales eran: organizar todo lo referente a los concursos anuales de atletismo, a los que eran llamados todos los habitantes del país, fomentar la construcción de plazas de deportes, fundar asociaciones deportivas, relacionarlas entre sí y con las extranjeras, recabar de las autoridades y del sector privado donativos para el fomento del deporte. (Forero, 1989, p. 352)
}

En la exposición de motivos para la aprobación de la Ley 80 de 1925, el defensor del proyecto y representante a la Cámara, Carlos Uribe Echeverri (citado por Forero, 1989, p. 352) esgrimió uno de los argumentos más potentes para su posterior aprobación al afirmar que: 
En nuestros días [1925] los deportes que se cultivan en Suecia nos han enseñado los efectos extraordinarios en la regeneración de ese país por medio de los ejercicios científicamente dirigidos. La organización gimnástica de los alemanes ha tenido una influencia poderosa en todo lo que en esa raza es unidad, fuerza, orden, disciplina. En Inglaterra los deportes han fijado también las características del pueblo y así lo dice la expresión consagrada: en los partidos de fútbol, de rugby de Eton, fueron desarrollados el valor y la tenacidad que cambiaron en Waterloo la derrota por la victoria.

Estas afirmaciones tendrán eco en algunos intelectuales de la época, como se expone a continuación.

Uno de aquellos intelectuales de la época es Miguel Jiménez López, quien aportó al debate de la época con la siguiente afirmación que va en relación directa con la cita del párrafo anterior: "tres son los métodos de educación física que en diversas épocas se han disputado el favor de los educadores: el método alemán o gimnástica con aparatos; el método inglés, o de los deportes; y el método sueco, llamado también gimnasia científica o racional" (Jiménez López, 1928, p. 235).

Los tres métodos mencionados, se adoptaron en Colombia. Los métodos alemán y sueco se aplicaron en el Gimnasio Moderno de Bogotá a partir de 1927, cuando se contrató al profesor Hans Huber, integrante de la Misión Pedagógica Alemana, quien a su vez, era un maestro que ofrecía cursos de educación física a otros maestros de Bogotá. Estos últimos asimilaron sus enseñanzas sobre ejercicios con aparatos, a mano libre, carreras, saltos y lanzamientos, con los cuales se podía formar un cuerpo capaz de enfrentar los avatares del mundo moderno. Una de las mayores dificultades para la difusión e implementación de este método en Colombia giraba en torno al elevado costo de los aparatos necesarios para la formación del cuerpo.

El método inglés ha tenido, hasta el día de hoy, una fuerte acogida en la formación del cuerpo desde la educación física en las escuelas, pero también desde la idea de un cuerpo sano que se logra a partir de la práctica de algún deporte, recomendado por médicos y educadores físicos. Este método es quizás el más pertinente para el desarrollo del capitalismo, pues los ideales de formación que persigue permiten en el sujeto educable, la incorporación de los valores centrales del espíritu capitalista, tales como el individualismo metodológico, utilitarismo, progresismo, interiorización de la norma, competición, entre otros.

Para los tres métodos mencionados se requiere de un entrenamiento permanente por parte de quien lo practica, y un guía o instructor que le permita el perfeccionamiento del ejercicio, a medida que repite las acciones necesarias para obtener el rendimiento requerido. Por eso, dentro del espíritu del método deportivo propuesto por los ingleses, se genera la necesidad de un entrenamiento 
deportivo guiado, apoyado en quienes, de manera inicial y empírica, serían los primeros entrenadores deportivos, que por lo general tenían relación directa con el ejército o habían tenido la oportunidad de practicar algún deporte, basándose en una transmisión empírica de sus conocimientos a sus discípulos.

Como respuesta a dos necesidades fundamentales del momento, resultaba imperativo pensar en un proyecto de reconciliación Nacional, asimismo, en el país se estaba presentando un perfeccionamiento y elevado nivel de rendimiento competitivo, además de los avances en el ámbito internacional, de ahí que fuera sancionada la ley 80 de 1925 mediante la cual se dio inicio a los primeros Juegos Nacionales. La práctica deportiva regular y sistemática de la población fueron determinantes para este proceso. Las primeras categorías que se dieron en las justas fueron la colegial y la particular, a razón de que los colegios y universidades tenían una fuerte representación en la competencia.

Los primeros Juegos Olímpicos Nacionales se organizaron en 1927, mediante el Decreto 1842, en Bogotá. Pero como caso curioso, este certamen pasó -después de haber finalizado-a ser considerado como un ensayo, el 26 de marzo de 1928, Miguel Abadía Méndez expidió el Decreto 560 que le dio vida a los Juegos Nacionales y le adjudicó la sede a la ciudad de Cali y no a Bogotá, como pretendían sus dirigentes, todo por el enfrentamiento entre el Gobierno Nacional y la Asociación Deportiva Colombiana que tenía el manejo directo del certamen.

Los deportes que hicieron presencia en las primeras justas deportivas fueron: ajedrez, atletismo, ciclismo, natación, boxeo, baloncesto, triciclo y fútbol. Así mismo, los departamentos considerados como pioneros fueron: Antioquia, Atlántico, Boyacá, Caldas, Cundinamarca, Huila, Magdalena, Norte de Santander, Santander, Tolima y Valle.

Queda marcada y muy visible la necesidad de los instructores, entrenadores o guías, como quieran llamarse a las personas encargadas de buscar el máximo rendimiento de los sujetos. Ya desde antes de 1925 se venían desarrollando procesos que intentaban mejorar los desempeños en competencia, sin embargo, no se tenía aún configurado un espacio competitivo en el cual se pudiera desarrollar, y es en este punto en el que radica la importancia de los Juegos Olímpicos Nacionales, como se les llamó en un principio.

La historia de los Juegos marcha de la mano con la del rendimiento deportivo porque se presenta una política de Estado para que los departamentos preparen deportistas y compitan en los juegos, implica esto la cualificación del personal técnico encargado de potencializar a los deportistas, pero también la revisión de los métodos que hasta entonces se venían aplicando. Como se expuso con anterioridad, se pensaba toda una estrategia mediada por el deporte en pos de 
la reconciliación Nacional. Producto de este proceso, Colombia participó en los Juegos Olímpicos de 1932 por primera vez con un deportista que asumió el reto de forma personal, sin el apoyo del Estado. Sin embargo, en 1936 se creó el Comité Olímpico Colombiano (COC) y ese año, Colombia se afilió al Comité Olímpico Internacional (COI) y participó en los Juegos Olímpicos con tres deportistas.

Como ya lo enunciamos antes, en el contexto internacional, por este mismo período, en Europa -especialmente Inglaterra, Francia y Alemania- se conforman clubes deportivos con fines higienistas. Es en este contexto en el que nacen algunos de los populares deportes que en la actualidad acaparan la atención de los espectadores. No está de más indicar que esta actividad física propuesta como remedio a lo que parecía una juventud decadente en unas sociedades igualmente decadentes, produjo el espíritu olímpico que aún pervive (cf. Burrow, 2001).

El atleta que quiere tener músculos poderosos acude al movimiento y al esfuerzo; la persona que desea adquirir la maestría en un ramo cualquiera de arte, una y mil veces empuña el pincel o se pone ante el teclado para ejercitar sus manos y sus sentidos. Así, en la educación del carácter: nuestro propósito es obtener hombres de voluntad firme y de personalidad definida? [sic] Pues debemos, desde sus primeros pasos, ejercitar a ese sér [sic] humano en el objeto único y específico de la voluntad: en la acción. (Jiménez López, 1928, p. 205)

En nuestro ámbito, este movimiento lo representaron personas como Miguel Jiménez López, Rafael Bernal Jiménez, Felipe Lleras Camargo, Eduardo Vasco (Padre), Gabriel Anzola Gómez, Tomás Rueda Vargas, Norberto Solano, Tomás Cadavid, Alfonso Jaramillo, Agustín Nieto Caballero, entre otros ${ }^{7}$. Además de las ideas que aquí se pusieron en juego, desde el punto de vista institucional y legislativo, también la Ley Uribe cumplió un papel determinante, según lo plantean Sáenz, Saldarriaga y Ospina (1997, cap. 8). De acuerdo con estos autores:

[la] Ley Uribe buscaba transformar los estudios teóricos en prácticos, enfatizando lo concreto sobre lo abstracto y lo real sobre lo ideal, y buscaba también remplazar "el sentimentalismo en la ciencia y la imaginación en las teorías", por estudios y trabajos escolares de utilidad económica. Así mismo, esta ley pretendía introducir el sistema educativo de los pueblos anglosajones, para poder lograr su nivel de progreso económico. Por otra parte, los métodos de enseñanza introducidos apuntaban a la superación del excesivo verbalismo y memorismo en que habían caído en la instrucción pública las Lecciones de Cosas [...] Esta reforma de los métodos debía basarse en las necesidades inmediatas del país, así como en las de los alumnos. En las escuelas primarias la escuela debía tener en cuenta la edad del alumno para no forzar su espíritu ni contrariar el "desarrollo natural de la razón", fomentando la enseñanza intuitiva y utilizando ejemplos relativos a su país y a su vida cotidiana, con el fin de despertar en él el espíritu de observación, de reflexión, de intención y de expresión. (Sáenz, Saldarriaga y Ospina, 1997, pp. 47-48)

7 Todos ellos también hacen parte de la Generación del centenario en la historia intelectual colombiana. 
Entre las funciones que debía cumplir la educación en la sociedad, estaba la misión de generar un elemento cultural integrador que limara las identidades regionales y aproximara a la población a una idea de nación que los identificara. Para el cabal cumplimiento de esta función se requería como condición necesaria, la unificación de los mensajes que debía transmitir el maestro, de manera tal que los docentes formados en las distintas regiones tuvieran las mismas calidades y recibieran el mismo tipo de formación. Esta necesidad se hace evidente en la Ley 6 de agosto de 1912 sobre el establecimiento de escuelas de primeras letras para los niños de uno y otro sexo.

Así pues, las pretensiones de un progreso y avance social en Colombia llevaron a que se viera la institución escolar como una parte estratégica para las pretensiones de homogenización de la población. Se trataba de que la pedagogía asumiera también el discurso del progreso y con base en él se diera a la tarea de posibilitar nuevas formas de subjetivación. En la práctica y en el discurso pedagógico, y por supuesto, en la escuela misma, radicaban pues, tanto las posibilidades de una educación "nueva", diferente, como las esperanzas de "progreso" de la sociedad. Así lo sostenía Miguel Jiménez López:

La humanidad está de tal manera constituida, que cada uno de sus miembros trae a la vida capacidades y dones llamados a proveer a cada una de las necesidades del conjunto social. A medida que en el transcurso de los tiempos la actividad humana, que en sus principios fue rudimentaria e instintiva, se ha ido diferenciando en mil direcciones que buscan sin cesar la perfección [... L La vida tiene ese milagroso poder de previsión que crea capacidades y poderes especializados allí donde son necesarios al mantenimiento y al avance de las especies. En las mismas especies que viven en colectividad, se advierte que hay individuos que nacen especialmente dotados para las funciones reproductoras, en tanto que otras traen aptitudes para la vida industrial de la sociedad, y, entre estos últimos, los hay organizados para dirigir, al paso que otros se adaptan por su conformación nativa a las humildes pero esenciales funciones del trabajo. (1948, pp. 77-78)

Desde finales del siglo XIX, la vida como referente formativo recibe interpretación por parte de pedagogías como las de Decroly y Dewey, quienes otorgan al médico la oportunidad de ponerse a la delantera de la formación de maestros. Es el momento en el cual la higiene se asume como instrumento de regeneración de la raza y la infancia empieza a mostrarse como un referente formativo autónomo. Es notable entonces, que desde fines del siglo XIX e inicios del siglo $\mathrm{XX}$, la apropiación progresiva del saber médico lograra popularizar una crítica, un malestar contra las condiciones físicas de la población pobre, y de manera especial, de la infancia que asistía a las escuelas oficiales.

De igual modo, como diría Catalina Reyes,

[...] en la Colombia de principios del siglo XX, la pobreza, el marginamiento [sic], la precariedad y el hacinamiento en las viviendas, la desnutrición, la falta de 
normas higiénicas y la ausencia de medidas de salubridad públicas hicieron de las ciudades un foco de enfermedades y epidemias que alcanzaban muchas veces cifras alarmantes de mortalidad. (1994, p. 15)

Es bajo este panorama que el discurso médico comenzó a ocupar un lugar irremplazable en la escuela y en los programas de la escuela para la población más pobre del país. Así, el médico cada día ocupaba más espacio en lo social, pues actuaba directamente en los consejos higiénicos y tratamientos de enfermedades en las escuelas, participaba en los debates realizados frente a las teorías pedagógicas y en los exámenes escolares. Como dato adicional y significativo, es importante indicar también, que la peste de gripe de 1918 en Santa Fe de Bogotá fue determinante para la hegemonización del saber médico e higienista en la 'cuestión social' del país (Noguera, 2003).

Además, el dispositivo de la medicalización fue muy eficiente en el sentido de delimitar los intereses públicos y los fines sociales, porque pasó a ser una necesidad nacional. De esta manera, en la década de 1920, la medicalización se aproximó como si fuese una extensión de la obra cristiana, lo cual evitó su rechazo en tanto que peligro para el dominio eclesiástico sobre las familias, que a pesar de todo, siguió siendo hegemónico. Qué mejor manera para iluminar lo dicho que con una cita del doctor Vasco, tomada de su Breviario de la madre (1934):

Porque todas estas fuerzas vitales, estas energías biológicas sólo [sic] tienen un valor relativo, frente a las energías que iluminan la vida superior del hombre, y que forman la espuma de la verdadera cultura. El torbellino de los días que corremos nos está mostrando con caracteres de tragedia, hasta dónde puede llegar el hombre máquina que no tiene más aspiraciones que el 'espacio vital' ni más ley que el derecho de conquista.

Lo anterior quiere decir que la fisiología y la nutrición no valen nada -y a veces menos que nada, muchas veces- si no están precedidas por la unidad que entrañan los factores morales, y que esta unidad sí valorizará hasta el infinito el enigma de los ceros, cuyo valor depende siempre de los guiones que los preceden o los siguen.

El magno error de estos tiempos ha sido sin duda el estímulo de una civilización que olvidó los factores espirituales, y que pasó por alto el cultivo de las necesidades de orden moral que son las únicas capaces de contrarrestar la natural elación del egoísmo humano. (1934, p. 221)

Posteriormente, en la década de los treinta, también se observó que los saberes biológicos como la medicina y la psicología, eran insuficientes para dar cuenta de toda la dinámica poblacional, y es entonces cuando se apropiaron saberes específicamente sociales, como la antropología y la sociología, trayendo como consecuencia, la exigencia de nuevos conocimientos al maestro. La con- 
secuencia en la práctica pedagógica en esta década consistió esencialmente en lograr que la escuela, el maestro y el niño fueran reproductores de una cultura higiénica basada en un saber higiénico, fisiológico y anatómico. Es entonces desde el discurso médico que en Colombia se introduce la pedagogía activa, ya que desde ahí se estudiaba la salud del cuerpo, de la mente y de la moral. Tal modelo lo adoptaron las escuelas normales a principios del siglo XX y se generalizó posteriormente a mediados de la década de 1930.

A propósito de la biopolítica, se pueden desarrollar hipótesis que van de la mano con las transformaciones sociales y culturales que se irán presentando de manera paulatina en Colombia, tal como sucede con la industrialización y su relación con el cuerpo en formación para el trabajo; la formación de la mujer y su cuerpo, a propósito de una mirada funcionalista y moral; la niñez y la tensión entre los tiempos para el estudio y los tiempos para el trabajo; la ubicación social de los niños y las personas "especiales", "discapacitados", "anormales"; la homosexualidad como "enfermedad"; la raza y etnia como categorías hegemónicas para la distribución socioeconómica de la población; entre otros. Pero esos pueden ser temas para otros escritos, que abandonamos en este caso para no perdernos en el bosque de las ideas.

\section{Conclusiones}

Es importante destacar que, inicialmente en Colombia el concepto de pedagogía activa implicaba un incremento en el movimiento físico del alumno. Dicha comprensión restringida cobró fuerza al articularse rápidamente al discurso médico de regeneración y vigorización fisiológica de la raza, y a las nuevas concepciones sobre la acción recíproca entre cuerpo, inteligencia y moral, como se daba en Europa en ese momento. De alguna manera, dicha propuesta iba en contra del modelo de educación moral y católica que dominaba para ese entonces. Estos cambios se dieron tanto en términos teóricos y prácticos como institucionales, cambios que resultaron de la progresiva apropiación de los saberes y conocimientos modernos, y de la recepción de los planteamientos del movimiento de pedagogía reformista o activa que por esa época se desarrollaba también en gran parte del mundo.

De otro lado, la aproximación a la comprensión de la formación discursiva denominada "entrenamiento deportivo", pone sobre la mesa una serie de enunciados dispersos que se van aglutinando y legitimando por aquellos intereses educativos y culturales que comienzan a emerger gracias a la preocupación por la degeneración de la raza y a otras alternativas que permitieron el progreso de la población colombiana durante la primera mitad del siglo XX, y que modificaron las condiciones biopolíticas para el ejercicio de poder sobre los cuerpos de la 
población, a través de la educación física como estrategia escolar y posteriormente, con el entrenamiento deportivo como estrategia social.

De esta manera, el entrenamiento deportivo se convierte en un instrumento fundamental para lograr fines y registros, representados en marcas, records, puntajes, entre otros, que se proponen para dar cuenta de los incrementos de fuerzas corporales y mayor capacidad de trabajo, con ello se le da vida a una elite deportiva. Así, el entrenamiento se configura como medio para generar economía fisiológica y estructural, soportada sobre un minucioso control de los rendimientos.

A su vez, con las diferentes etapas en la evolución del entrenamiento deportivo, se presentan dos líneas que llevan a significados diferentes. La primera tiene un alto compromiso con la estructura y planificación de las tareas que buscan el máximo aprovechamiento de las capacidades energéticas, la relación estimulo - descanso manifiesta la habilidad para la producción de esfuerzos en el sujeto. La segunda, tiene que ver con el proceso de industrialización, aparece el tema de la estética como elemento diferenciador, el cual prevalece sobre la fuerza y la destreza. La higiene y la apariencia exterior del cuerpo se asocian a la salud, al bienestar y a la acción lúdica del cuerpo.

\section{Referencias}

Álvarez, J. (2012). Educación, progreso y cuerpo en Colombia entre 1920 y 1940: el caso de Antioquia. En A. Runge y B. Escobar (Comp.) (2012), Educación, eugenesia y progreso: biopoder y gubernamentalidad en Colombia (pp. 236). Medellín: Edición Unaula.

Angulo, A. M. (2007). Conceptos pedagógicos alemanes en la educación colombiana: La Segunda Guerra Mundial y la actualidad. Matices en lenguas extranjeras, 1.

Arboleda, R. (1992). Una aproximación entre la antropología y la educación física. Educación física y deportes, 14, 81-91.

Betancur, G. (1984). Presentación Ley 39 de 1903. Revista Colombiana de Educación, (13), 121-135.

Bohórquez Casallas, L. A. (1956). La evolución educativa en Colombia. Bogotá: Cultural Colombiana.

Bompa, T. (2007). Periodización. Teoría y metodología del entrenamiento (2ºd.). Barcelona: Editorial H. Europea.

Burrow, J. (2001). La crisis de la razón, el pensamiento europeo, 1848 - 1914. Barcelona: Crítica.

Cacua Prada, A. (1997). Historia de la Educación en Colombia. Santa Fe de Bogotá: Academia Colombiana de Historia.

Castro, E. (2004). El vocabulario de Michel Foucault. Buenos Aires: Universidad Nacional de Quilmes.

Castro, J. (2008). Constitución política de Colombia ( $2^{\circ}$ ed.). Bogotá: Editorial Universidad del Rosario.

Castro, J. J. (1938). Educación nacional. Informe al Congreso. Bogotá: Editorial ABC. 
Chinchilla, V. J. (1999). Historiografía de la educación física en Colombia. Revista Colombiana de Educación, (38-39), 101-116.

Congreso de Colombia (1991, enero 18). Ley 181 de 1995. Ley del deporte. Por el cual se dictan disposiciones para el fomento del deporte, la recreación, el aprovechamiento del tiempo libre y la Educación Física y se crea el Sistema Nacional del Deporte. Diario Oficial (41.679). Recuperado de https://www. mineducacion.gov.co/1621/articles-85919_archivo_pdf.pdf

Cortés Díaz, J. T. (2013). Análisis en torno a la relación entrenamiento deportivo - formación de sujeto deportista desde perspectivas críticas latinoamericanas: caso Indeportes Antioquia 1996 - 2011. (Tesis de maestría). Universidad San Buenaventura, Medellín. Recuperado de http://hdl.handle.net/10819/1649

Cortes Díaz, J. T. (2015). Deporte y posconflicto: una opción para la convivencia. En S. N. Cogollo Ospina (coord.), Imaginar la paz en Colombia. cavilaciones desde la academia (pp. 72-86). Medellín: Fundación Universitaria Luis Amigó.

Forteza de la Rosa, A. (1999). Direcciones del Entrenamiento Deportivo. Metodología de la preparación del deportista. La Habana, Cuba: Ed. Científico Técnica.

Forero, M. (1989). El deporte en Colombia. En Á. Tirado Mejía, Nueva Historia de Colombia. Vol. 6 (pp. 399), Bogotá: Planeta Colombiana Editorial.

Gallego, F. y Cano, E. (1988). Coldeportes Antioquia: 20 Años Promoviendo en el Departamento. Educación física y deportes, 10, 64-70.

Giddens, A., y Fehér, F. (1995). Biopolítica. La modernidad y la liberación del cuerpo. Barcelona: Península.

González Acevedo. E. (2006). ¿Es el deporte, la recreación y la educación física en Colombia, un derecho fundamental? (Trabajo de Especialización). Universidad de Antioquia, Medellín. Recuperado de: http:// viref.udea.edu.co/contenido/pdf/039-deportederecho.pdf

González, M. E. (2010). Sociedad y deporte: análisis del deporte en la sociedad y su reflejo en los medios de comunicación en España. España: Universidad de la Coruña.

González, R. Madera, E., y Salguero, A. (2004). Las escuelas gimnásticas y su relación con la actividad física y educación física actuales. Efdeportes, 10(73). Recuperado de https://www.efdeportes.com/ efd73/gimn.htm

Harre, D. (1987). Teoría del entrenamiento deportivo. Buenos Aires: Editorial Stadium.

Helg, A. (2001). La educación en Colombia, 1918 - 1957. Una historia social y económica (2ed.). Bogotá: Universidad Pedagógica Nacional.

Le Bot, I. (1979). Educación e ideología en Colombia. Medellín: La Carreta.

López, M. J. (1928). La escuela y la vida. Impr. réunies. Fundación Confiar.

Jiménez, M. (1948). La actual desviación de la cultura humana. Discursos y ensayos. Tunja: Imprenta Oficial.

Machado, H. y Rodas. S. (1983). Educación, deporte y psicología. Medellín: Politécnico Colombiano.

Matveiev, L. P. (1982). El proceso del entrenamiento deportivo. Argentina: Editorial SRL Stadium.

Matveiev, L. P. (1977). Principios del entrenamiento deportivo. Moscú: Cultura Física y Deporte.

Matveiev, L. P. (2001). Teoría general del entrenamiento deportivo. Barcelona: Editorial Paidotribo.

- 108 
Martin, D., Carl, K., y Lehnertz, K. (2001). Manual de metodología del entrenamiento deportivo. Barcelona: Editorial Paidotribo.

Mendoza, W. (2009). Consideraciones teóricas para el desarrollo de estudios sociohistóricos del deporte. Cuaderno Venezolano de Sociologia, 18(1), 45-64.

Mesa, E., A. (1985). Aproximación al estudio de la nueva legislación sobre deporte, educación física y recreación (ley marco). Educación física y deportes, 7-11.

Ministerio de Educación Nacional (MEN) (1959). Educación Colombiana. disposiciones orgánicas y reglamentarias de la educación nacional de 1903 a 1958. Bogotá: Imprenta del Ministerio de Educación Nacional.

Molano, A., y Vera, C. (1984). Evolución de la política educativa durante el siglo XX, primera parte, 1900 - 1957. Revista Colombiana de Educación, (13), 139-143.

Muñoz, D., y Alvarado, S. (2009). La integralidad como multidimensionalidad, un acercamiento desde la teoría crítica. Hologramática, 1, 103-116.

Muñoz, D.A., Muñoz, E.A., y García, L. (2010). El desarrollo humano y la pedagogía: diálogo de discursos. Acierto: Revista académica y científica, (1657-5954), 9-15.

Muñoz, C. (2011). Los problemas de la raza en Colombia. Más allá del problema racial: el determinismo geográfico y las "dolencias sociales". Bogotá: Universidad del Rosario, Memoria Viva Colección Bicentenario.

Noguera, C. (2003). Medicina y política: discurso médico y prácticas higiénicas durante la primera mitad del siglo XX en Colombia. Medellín: Fondo Editorial Universidad Eafit.

Ozolín, N. (1970). El sistema moderno de entrenamiento deportivo. La Habana: Educación Física y Deporte.

Ozolin, N. G. (1983). Sistema contemporáneo de entrenamiento deportivo. La Habana, Cuba: Editorial Científico Técnica.

Parent, J. (1990). Para una ética del deporte. (M. de Navarrete, Ed.). Michoacán: Julián H Guaraldo.

Pérez, J. y Pérez, D. (2009). El entrenamiento deportivo: conceptos, modelos y aportes científicos relacionados con la actividad deportiva. Revista digital Efdeportes, 13.

Prieto, V. (2000). El Gimnasio Moderno y la formación de la élite liberal bogotana, 1914 - 1948. Bogotá: Universidad Pedagógica Nacional.

Quitián Roldán, D. L. (2013, enero-junio). Deporte y modernidad: caso Colombia. Del deporte en sociedad a la deportivización de la sociedad. Revista Colombiana de Sociología, 36(1), 19-42.

Rauch, A. (1985). El cuerpo en la educación. Buenos Aries: Editorial Kapelusz.

Reyes, C. (1994, junio). Higiene y salud en Medellín, 1900 - 1930. Estudios Sociales, (7), 13-43.

Ruiz Patiño, J. H. (2009). La política del sport: elites y deporte en la construcción de la nación colombiana, 1903-1925. (Tesis de Maestría). Pontificia Universidad Javeriana, Bogotá. Recuperado de http:// repository.javeriana.edu.co/bitstream/10554/343/1/pol96.pdf

Runge, A, Muñoz, D., Álvarez, J (2005). Degeneración de la raza y necesidad de una cultura/educación física: cuerpo y evolucionismo social en la Colombia de la primera mitad del siglo XX. Revista Itinerario Educativo, $\operatorname{XIX(46),~} 124$. 
Runge, A, Muñoz, A. (2012). Evolucionismo social, problemas de la raza y educación en Colombia, primera mitad del siglo XX: el cuerpo en las estrategias eugenésicas de línea dura y línea blanda. Medellín: Universidad Autónoma Latinoamericana.

Sáenz Obregón, J., Saldarriaga, O., y Ospina, A. (1997). Mirar la infancia: pedagogía, moral y modernidad en Colombia, 1903 - 1946. Colombia: Colciencias, Ediciones Foro Nacional por Colombia, Ediciones Uniandes, Editorial Universidad de Antioquia.

Vasco, E. (1934). Breviario de la madre. Medellín, Bedout.

Vélez, B. (1993). Sociología del deporte: algunos problemas teóricos y epistemológicos. Educación Física y Deporte, 15(1), 65-72.

Verjoshanski, I. V. (1982). Principios del entrenamiento para atletas de élite. Moscú: Academia de Ciencias de la URSS. 